S2 Table. Database search strategies

PubMed

\begin{tabular}{|l|l|}
\hline 1. Pertussis vaccine & 2. Pregnant women \\
Pertussis vaccine [majr] OR & $\begin{array}{l}\text { Pregnant women [majr] OR } \\
\text { Pertussis vaccin*[ALL] OR } \\
\text { Phooping cough }\end{array}$ \\
vaccin*[ALL] OR & Maternal* [ALL] \\
Diphtheria-Tetanus-acellular & \\
Pertussis Vaccin* [ALL] OR & \\
DTaP Vaccin* [ALL] OR & \\
Diphtheria-Tetanus-Pertussis & \\
Vaccin*[ALL] & \\
\hline
\end{tabular}

(Pregnant women [MH] OR Pregnan*[ALL] OR Maternal* [ALL]) AND (Pertussis vaccine [MH] OR Pertussis vaccin*[ALL] OR Whooping cough vaccin*[ALL] OR DiphtheriaTetanus-acellular Pertussis Vaccin* [ALL] OR DTaP Vaccin* [ALL] OR DiphtheriaTetanus-Pertussis Vaccin*[ALL) NOT (ANIMALS[MH] NOT Humans[MH])

\title{
1 AND 2
}

Hits $=\mathbf{5 7 8}$

Filter $=$ Pub dates 10 years

Hits $=350$

PMC

(Pregnant women [majr] OR Pregnant[ALL] OR Pregnancy[ALL] OR Maternal [ALL]) AND (Pertussis vaccine [majr] OR Pertussis vaccin*[ALL] OR Whooping cough vaccin*[ALL] OR Diphtheria-Tetanus-acellular Pertussis Vaccin* [ALL] OR DTaP Vaccin* [ALL] OR Diphtheria-Tetanus-Pertussis Vaccin*[ALL) NOT (ANIMALS[MH] NOT Humans[MH])

$$
\text { Hits }=1631
$$

Filter $=$ Pub dates 10 years

Hits $=865$

Medline- Ovid

1. exp *Pregnant women/

2. Pregnan\$.MP.

3. Maternal\$ .MP.

4. 1 or 2 or 3 AND

5. exp *Pertussis vaccine/ 
6. Pertussis vaccin\$.MP.

7. Whooping cough vaccin\$.MP.

8. Diphtheria-Tetanus-acellular Pertussis Vaccin\$ .MP.

9. DTaP Vaccin\$ .MP.

10. Diphtheria-Tetanus-Pertussis Vaccin\$.MP.

115 or 6 or 7 or 8 or 9 or 10

12. 4 and 11

13 limit 12 to $y r=$ "2006 -Current"

14. Humans/

15. exp animals/

16.15 NOT 14

17. 13 NOT 16

Hits $=1946$

CINAHL

\begin{tabular}{|l|l|}
\hline$\#$ & Query \\
\hline S13 & S9 AND S12 \\
\hline S12 & $\begin{array}{l}\text { (MM "Pertussis Vaccine") OR TX "Pertussis vaccin*" OR TX "Whooping cough } \\
\text { vaccin*" OR TX "DTap vaccin*" }\end{array}$ \\
\hline S11 & (MM "Pertussis Vaccine") \\
\hline S10 & (MH "Pertussis Vaccine+") \\
\hline S9 & (MM "Expectant Mothers") OR TX Pregnan* OR TX Maternal* \\
\hline S8 & (MM "Expectant Mothers") \\
\hline S7 & S2 AND S5 \\
\hline S6 & S2 AND S5 \\
\hline S5 & $\begin{array}{l}\text { (MM "Pertussis Vaccine") OR TX "Pertussis vaccin*" OR TX "Whooping cough } \\
\text { vaccin*" OR TX "DTap vaccin*" }\end{array}$ \\
\hline S4 & (MM "Pertussis Vaccine") \\
\hline S3 & (MH "Pertussis Vaccine+") \\
\hline S2 & (MM "Expectant Mothers") OR TX Pregnan* OR TX Maternal* \\
\hline S1 & (MM "Expectant Mothers") \\
\hline
\end{tabular}

Hits $=381$

Combined hits after removing duplicated articles on Endnotes $=1935$

\section{Other Sources}

I. European Society for Paediatric Infectious Diseases (ESPID)

Abstracts from 2011- 2016 
Hits $=0$

II. World Society for Paediatric Infectious Diseases (WSPID)

WSPID 2015 and WSPID 2013 and WSPID 2011

Hits $=0$

III. International Congress on Infectious Diseases (ICID) $=0$

Total hits $=1935$ 